\title{
Neutrino Oscillations: Experimental Review
}

\author{
Anatael Cabrera* \\ CNRS/IN2P3 - APC Laboratory \\ E-mail: anatael@in2p3.fr
}

The Neutrino Oscillation phenomenon has been fully established manifestation of both the nontrivial mixing in the leptonic sector, characterised by the PMNS ( $3 \times 3$ matrix in the 3 neutrino type scenario) and the non-degenerate mass state spectrum of neutrinos. As a consequence, we know that mixing is part of the phenomenology of both quarks and leptons, including $\mathrm{CP}$ violation, although their mixing patterns have so far been measured to be very different and the possible physics behind remains unknown. Evidence for neutrino $\mathrm{CP}$ violation has not yet been observed, due to lack of experimental sensitivity. Also, thanks to the observation of neutrino oscillations, neutrinos are known to be massive. The absolute mass value is still unknown, being much lower than all other known fermions. In my talk, I will briefly review the experimental status of our knowledge as of the Neutrino 2014 (June 2014 at Boston, USA) conference. Improvements due to foreseen experiments will be also highlighted.

Frontiers of Fundamental Physics 14 - FFP14,

15-18 July 2014

Aix Marseille University (AMU) Saint-Charles Campus, Marseille

${ }^{*}$ Speaker. 